\title{
RESÚMENES DEL I CONGRESO INTERNACIONAL DE LA SOCIEDAD DE LA NEUROCIENCIA PARA EL PERÚ (SONEP)
}

\author{
Editores: Luis Angel Aguilar Mendoza, Vanessa Minaya Lescano, \\ Nazareth Carigga Gutierrez, Jenny Quezada Zevallos
}

La Sociedad de la Neurociencia para el Perú (SONEP) es la máxima entidad rectora de la Neurociencia en el Perú y está adscrita desde el 2003 a la International Brain Research Organization (IBRO), entidad mundial que agrupa a 64 países en el campo de la Neurociencia. La SONEP es una organización creada sin fines de lucro, fundada en el 2003, ante la necesidad de los científicos peruanos, de contar con un apoyo interdisciplinario y transparencia académica en el inicio, desarrollo y actualización de las ciencias que estudian de manera integral el sistema nervioso y su manifestación hacia el medio externo. Esta sociedad está conformada por profesionales de diversas disciplinas que estudian el sistema nervioso desde términos moleculares hasta comportamentales. Convoca investigadores básicos y clínicos de variados centros de investigación y docencia del Perú.

El I Congreso Internacional de la Sociedad de la Neurociencia para el Perú (SONEP) se llevó a cabo del 24 al 28 de octubre del 2017, estuvo a cargo del comité organizador responsable de dirigir el Congreso, en cooperación con las entidades: IBRO (International Brain Research Organization), Universidad Peruana Cayetano Heredia Universidad Femenina del Sagrado Corazón y Universidad San Ignacio de Loyola. El evento estuvo dirigido a investigadores, profesionales y estudiantes vinculados a carreras de la salud (Médicos especialistas, generales y residentes; tecnólogos médicos, psicólogos, biólogos, veterinarios), carreras sociales y de letras (Marketing, educación) así como al público en general interesado en conocer los avances y trabajos realizados en el ámbito de las neurociencias.

La neurociencia como disciplina interdisciplinaria ofrece múltiples posibilidades de desarrollo en múltiples especialidades (medicina, psicología, biología, ingenierías, educación, entre otros) que aportan en el estudio del sistema nervioso e implica el logro de competencias y su aplicación en trabajos de investigación. Diversos estudios asociados a esta disciplina promueven el desarrollo de nuevas herramientas para que sea posible el entendimiento y mejora de diversas patologías neurodegenerativas.

Las actividades y trabajos que se realizan en este campo son los siguientes: neurociencia y comportamiento, neurociencia molecular, inmunohistoquímica, modelos experimentales de enfermedades neurodegenerativas (Parkinson, Alzheimer y Epilepsia, expresión de neuropéptidos en el sistema nervioso central, acción neuroprotectora de plantas medicinales sobre el sistema nervioso central), neurociencia aplicada al marketing, así como varios estudios que vinculan el estudio del cerebro y sus modificaciones de comportamiento.

El objetivo de este I Congreso Internacional de la Sociedad de la Neurociencia para el Perú (SONEP) 2017 fue difundir de manera teórica y práctica diversos temas de vanguardia vinculados a la Neurociencia, así como incentivar el vínculo de científicos, investigadores, profesionales y estudiantes interesados en los trabajos que esta disciplina realiza. Además de difundir propiamente la investigación emanada de esta disciplina.

\section{CONFERENCIAS}

\section{SISTEMA SUBSTANCIA P/RECEPTOR NEUROQUININA-1 Y CÁNCER \\ Coveñas, R. (Instituto Neurociencias de Castilla y León (INCYL), Universidad de Salamanca. Salamanca, España).}

Muñoz, M. (Hospital Universitario Virgen del Rocío. Sevilla, España)

Introducción: El péptido sustancia $\mathrm{P}(\mathrm{SP})$ tras unirse al receptor neuroquinina-1 (NK-1) favorece la mitogénesis de las células tumorales. 
Objetivo: Estudiamos la acción antitumoral (mediante apoptosis) de los antagonistas no peptídicos del receptor NK-1 (incluido el antiemético utilizado en clínica, aprepitant) sobre numerosas líneas celulares tumorales humanas (neuroblastoma, glioma, retinoblastoma, mesotelioma, cáncer de pulmón, páncreas y mama, hepatoblastoma, osteosarcoma).

Procedimiento: En total hemos estudiado 25 líneas celulares humanas tumorales pertenecientes a 16 tipos de cáncer. Estos experimentos se han realizado tanto in vivo como in vitro.

Resultados: Cualquier línea celular tumoral humana se comporta de la misma manera ante la SP y los antagonistas del receptor NK-1: 1) La SP ejerce una acción mitogénica en dichas células y favorece la angiogénesis y la metástasis. Hemos demostrado la expresión de SP y del receptor NK-1 en los tumores estudiados. 2) El receptor NK-1 está sobre expresado en las células tumorales. 3) El receptor NK1 interviene en la viabilidad de las células tumorales. 4) Los antagonistas del receptor NK-1 ejercen efectos contrarios a los ejercidos por la SP: acción antitumoral (induce apoptosis en las células tumorales. Esta acción es dosis dependiente); efecto antiangiogénico (impide la formación de vasos sanguíneos) y efecto antimetástasis.

Conclusión: Los resultados obtenidos indican que el receptor NK-1 es una diana terapéutica para el tratamiento del cáncer. Los resultados apoyan la realización de un ensayo clínico que compruebe la acción antitumoral, antiangiogénica y antimetástasis de los antagonistas del receptor NK-1 en general y concretamente del aprepitant.

\section{EJERCICIO FÍSICO Y SU IMPACTO SOBRE EL SISTEMA NERVIOSO; DE LA EVIDENCIAA LA APLICACIÓN EN SALUD}

Riqueme-Uribe, D. . ${ }^{1,{ }^{3}}$ Navarro, P.; Cartes, E. .; Torres, V. ${ }^{3}$; Juica B ${ }^{3}$; Peiret L. ${ }^{3}$, Cofré C. ${ }^{3}$; Farías-Valenzuela, C. ${ }^{3,4}$

1. Centro de Investigación, Desarrollo e Innovación APLICAE - Chile.

2. Laboratorio de Neurociencia y Comportamiento; Universidad Peruana Cayetano Heredia - Perú.

3. Facultad de Ciencias Médicas,
4. Escuela de Ciencias de la Actividad Física, el Deporte y la Salud, Carrera de Terapia en Actividad Física y

Salud, Universidad de Santiago de Chile - Chile

Introducción: El propósito de esta conferencia, fue plantear un panorama sobre las evidencias que actualmente existen en la investigación, en relación al impacto del ejercicio físico sobre el sistema nervioso humano, con el fin de asociarlas con sus potenciales aplicaciones en salud y calidad de vida.

La evidencia es consistente en asociar al ejercicio, con otros efectos beneficiosos sobre mecanismos neurofisiológicos en condiciones patológicas complejas como diabetes I y diabetes II, cáncer de mamá, disfunción renal y hemodiálisis, incluso en los casos de condiciones neurológicas y neurocognitivas de gran complejidad (como síndrome de Down y Asperger) en los estudiantes chilenos con los que se está implementando el programa "inclusión en movimiento".

En este sentido, las evidencias levantadas a través de revisiones sistemáticas nos ayudaron a plantear los efectos agudos (como las modificaciones en el flujo sanguíneo cerebral y el aumento en plasma de BDNF y Cortisol) y las adaptaciones crónicas (como la neuroplasticidad y los cambios de conducta) inducidas por el ejercicio físico sobre el sistema nervioso. Dichos cambios son clave para el uso del ejercicio como una estrategia para el trabajo con pacientes de diversas condiciones.

Conclusión: En conclusión, basados en la evidencia actual disponible, el ejercicio físico debe ser considerado como parte del tratamiento complementario de condiciones neurofisiológicas, neuropatológicas y neurocognitivas, siempre y cuando se cuente con un profesional experto (terapeuta en actividad física y salud, profesor de educación física, entre otros) que cuente con la especialización teórica y práctica necesaria, además del manejo de la evidencia de primera línea.

Agradecimientos: los resultados presentados en esta conferencia fueron obtenidos gracias a los siguientes Proyectos de la Universidad de Santiago de Chile: a) incubadora de iniciativas de aprendizaje y servicio del Proyecto de Armonización Curricular USA 1502 de la Vicerrectoría Académica, b) premio a la bi - direccionalidad de la Vicerrectoría de 
Vinculación con el Medio, c) proyecto DICYT opinión 442 de la Dirección de Investigación Científica y Tecnológico y d) apoyo a publicaciones científicas de la Vicerrectoría de Investigación. Los resultados presentados de los trabajos del Grupo de Estudio en Neurociencia; Cognición en Movimiento (GEN CeM) fueron obtenidos gracias a la colaboración del Centro de Investigación, Desarrollo e Innovación APLICAE con Epistemonikos y el Centro de Evidencia de la Universidad Católica de Chile.

\section{PONENCIAS}

\section{IN SILICO ANALYSIS OF CELL NUCLEI ON DIFFERENT CELLULAR STRESS MODELS}

Hernandez-Baltazar D. ${ }^{1}$, Heman-Bozadas $\mathbf{P}^{2}{ }^{2}$, Rosas-Jarquin C.j. ${ }^{3}$, Barrientos-Bonilla A. ${ }^{3}$, Pensado-Guevara $\mathbf{P b} .^{3}$, Sandoval-Vargas $\mathrm{Ca}^{3}{ }^{3}$, Nadella R. ${ }^{4}$, Zavala-Flores Lm. ${ }^{5}$ VillanuevaOlivo A. ${ }^{6}$

1. CONACYT-Instituto de Neuroetologia, Universidad Veracruzana. Xalapa, Veracruz. Mexico.

2. Posgrado en Neuroetologia, Universidad Veracruzana. Xalapa, Veracruz, Mexico.

3. Facultad de QuimicaFarmaceuticaBiologica. Universidad Veracruzana. Xalapa, Veracruz. Mexico.

4. Department of Biotechnology, Sri PadmavatiMahi laVisvavidyalayam, Tirupati; CONACyT-Instituto de Neuroetologia (International collaboration ID1840), India

5. Centro de InvestigacionBiomedica del Noreste. Monterrey, Nuevo Leo Mexico.

6. Facultad de Medicina. Universidad Autonoma de Nuevo Leon. Monterrey, Nuevo Leon. Mexico.

Introduction: The process of induction of cellular stress by diverse stimuli is accompanied by cellular processes such as inflammation, degeneration and cell death. Although there are various immunostaining techniques for diagnostic studies using haematoxylineosin, the analysis of neuronal nuclear patterns (or of somatic cells) has been neglected.

Objective: To determine nuclear morphometric patterns in neurons and in somatic cells on diverse stress models.

Procedures: In this work, cellular stress was induced by four different approaches: 1) intraperitoneal administration of lipopolysaccharide, an immune response activator, 2) intranigral injection of Staurosporine, an apoptosis enhancer, 3) partial hepatectomy in rats and 4) cytotoxicity assay in SHSY5Y cell lines. The tissue sections $(5-10 \mu \mathrm{m})$ or cells preparations (monolayer) were immunostained with Hoechst 33342 (nuclear marker), CD11b/cOX-42 (microglial marker), tyrosine hydroxylase (dopaminergic phenotype marker) and caspase-3 (apoptosis marker). To evaluate shape, size, number and hyper/hypochromic features, we used ImageJ software.

Results: During inflammation, degeneration and cell death processes, particulars regarding the nuclear patterns were distinguished.

Conclusion. Regardless of the stressor stimulus, the analysis of the nuclear patterns contributes to the histopathological diagnosis.

\section{INFLUENCIA DE LA MASTICACIÓN DEFICIENTE SOBRE LA MEMORIA Y EL APRENDIZAJE ESPACIAL}

Aguirre-Siancas, E. (Facultad de Medicina. Universidad Nacional Mayor de San Marcos. Lima, Perú. Facultad de Ciencias de la Salud. Universidad Científica del Sur. Lima, Perú).

Introducción: La memoria y el aprendizaje son funciones cardinales en todos los seres vivos. En los humanos el deterioro de dichas funciones tiene un impacto devastador en las personas, las familias y la sociedad. Muchas causas explican este deterioro, dentro de ellas está la masticación deficiente.

Objetivo: Determinar la influencia de la masticación deficiente sobre la memoria y el aprendizaje espacial.

Procedimiento: Estudio experimental realizado en 20 ratones albinos machos de 5 meses de edad de la cepa Balb/c. Los animales se dividieron en el grupo masticación normal y el grupo masticación deficiente en base a la modificación de la consistencia de la dieta. La evaluación se realizó en al séptimo mes de vida lo animales en la fase de adquisición y luego en la fase de recuperación de memoria y aprendizaje espacial en el paradigma de Morris. Se empleó la prueba t para muestras independientes y el nivel de significancia considerado fue de 0,05 . 
Resultados: Se halló diferencias estadísticas en el primer día $(\mathrm{p}=0,035)$ de la fase de adquisición a favor del grupo masticación normal. En el resto de días de dicha fase no hubo diferencias entre ambos grupos ( $\mathrm{p}>$ $0,05)$, aunque siempre el grupo masticación normal tuvo mejor performance. En la fase de recuperación no hubo diferencias.

Conclusión: La masticación deficiente influye negativamente en la formación de memoria y aprendizaje espacial en ratones albinos machos y adultos de la cepa Balb/c.

\section{MORALIDAD Y DESARROLLO MORAL DESDE LA NEUROCIENCIA SOCIAL}

Cárdenas Macher, L. (Universidad Peruana Cayetano Heredia).

Introducción: Los paradigmas de la neurociencia social, análogos a los presupuestos trabajados en la teoría de sistemas dinámicos no lineales, permite expresar los límites argumentativos y conceptuales, incluso epistemológicos, de los diversos trabajos que reducen el problema de la moral y del desarrollo moral, a lo largo de la vida, a un comportamiento pro-social o predominantemente utilitarista; así como también de trabajos que abordan el mejoramiento moral gracias al uso de oxitocina intranasal. Frente a ello, esta presentación abre la discusión en la materia y presenta una línea de investigación con un concepto delimitado para la dimensión "moral" y sugerencias para abordar la problemática.

Objetivo: Reconocer la contribución de la neurociencia social como herramienta de análisis interdisciplinario para comprender el desarrollo moral y su expresión más plena (intuición, juicio y acción moral) a partir de la interacción del cerebro encarnado con su contexto socio-ambiental. Esto permite analizar las implicancias de la evidencia neurocientífica en materia de arquitectura cerebral y sistemas complejos, psicología del desarrollo y educación moral.

Procedimiento: El presente trabajo ha sido el resultado de una investigación cualitativa, y específicamente, se trabaja desde la metodología denominada como "teoría fundamentada". Esta metodología permitió evaluar la consistencia de las teorías neurocientíficas aplicadas a explicar y comprender la dimensión moral.Para ello, se estableció un análisis riguroso de la evidencia científica y de los presupuestos epistemológicos de las teorías propuestas en la materia.

Conclusiones: Se define la dimensión moral como la expresión última de un conjunto de sistemas, normativos y de fisiología humana, que interactúan articuladamente para poder comprender situaciones problemáticas de convivencia, originadas por el dolor social, que expresan la necesidad de evaluar alternativas y tomar decisiones con sentimiento de independencia, obligación e impersonalidad. Esta definición es coherente con los siguientes principios de organización cerebral: articulación dinámica no lineal entre sistemas (nervioso, autónomo), sincronía y reutilización neural. Estos principios permiten comprender un desarrollo moral capaz de desplegar comportamientos autónomos en niños y no solo en jóvenes, así como fortalecer la capacidad de re-evaluación cognitiva para reorientar sentimientos morales no conscientes a través de fomentar la curiosidad, promover inquietudes y aplicar retroalimentación constante en la formación de niños, niñas y adolescentes.

\section{RELACIÓN ENTRE LOS RESULTADOS DE LA ESCALA DE MEMORIA DE TRABAJO Y LAS ONDAS THETA Y ALFA EN PACIENTES POST ACV}

Castro, M. J. (Universidad Peruana Cayetano Heredia)

Introducción: Relación entre los resultados obtenidos de la escala de memoria de trabajo y las ondas theta y alfa en pacientes luego de un accidente cerebro vascular, del área de neurología de un hospital nacional de lima.

Objetivos: Encontrar la relación entre los resultados obtenidos de la escala de memoria de trabajo y las ondas theta y alfa en pacientes luego de un accidente cerebro vascular, del área de neurología de un hospital nacional de Lima.

Procedimiento: Se escogieron a los pacientes, dependiendo del Mini Mental State Examination(MMSE), el cual determinó si el paciente es apto o no, para hacer las demás pruebas requeridas para esta investigación. Luego, se comenzó con el 
proceso de la investigación, donde se tomó el subtest de memoria de trabajo del WAIS IV, y además se le colocó EEG al paciente, mientras se tomaba la prueba psicológica, esto tomó un tiempo máximo de 20 minutos por paciente. Las señales del EEG son recolectadas con 9 electrodos, localizados en la posición estándar de 10/20 y 10/10 del sistema internacional. Los electrodos son puestos, sobre el cuero cabelludo del sujeto, con la ayuda de un EEG cap, en las posiciones: F8, AF8, Fp2, FPZ, Fp1, AF7, F7, O1 y O2, tomando como referencia el sistema 10/20 y 10/10.

Resultados: Se encontró que entre la escala de memoria de trabajo de WAIS IV y las ondas theta, hay una correlación Moderada Positiva, por lo que, al incrementar las puntuaciones de la escala de memoria de trabajo, la frecuencia de las ondas theta tiende a sincronizarse. Mientras que, en el caso de la relación entre las variables escala de memoria de trabajo de WAIS IV y las ondas alfa, se encontró que existe una correlación Inversa Baja, por lo que mientras que la puntuación de escala de memoria de trabajo sube, la frecuencia de las ondas alfa tiende a desincronizarse.

Conclusión: La memoria de trabajo se encuentra relacionada con la aparición de las ondas theta, mientras que las ondas alfa descienden cuando las puntuaciones de memoria de trabajo suben en el cerebro de pacientes post $\mathrm{ACV}$, al ser estudiadas por electroencefalografía.

\section{CAPACIDAD PROTECTORA DE LEPIDIUM MEYENII EN UN MODELO DE LA ENFERMEDAD DE ALZHEIMER}

Minaya, V., Pacheco, L. F. y Aguilar, L. A. (Laboratorio de Neurociencia y Comportamiento, Universidad Peruana Cayetano Heredia).

Introducción: La enfermedad de Alzheimer es una enfermedad neurodegenerativa considerada como la forma más frecuente de demencia, aproximadamente veinticuatro millones de personas padecen esta enfermedad. Se caracteriza principalmente por la pérdida progresiva de la memoria y deterioro de las funciones cognitivas. Una de las características histopatológicas de la enfermedad de Alzheimer es la formación de placas seniles, las cuales conducen a la formación de agregados proteicos como la proteína beta amiloide y la proteína tau. La proteína beta amiloide presenta actividad citotóxica debido a la producción de especies reactivas de oxígeno (ROS), los cuales tienen un efecto perjudicial en las poblaciones neuronales de la red septum hipocampo. Por ello, el suministro de sustancias con capacidad antioxidante podría revertir los efectos causados por la proteína beta amiloide. Lepidium meyenii, es una planta herbácea, alto andina y nativa del Perú con propiedades medicinales. Sin embargo, aún se desconoce su efecto neuroprotector sobre algunas patologías neurodegenerativas. Por ello, el presente estudio tiene como objetivo evaluar la capacidad neuroprotectora de Lepidium meyenii en un modelo animal de Alzheimer con daño por estrés oxidativo cerebral inducido por el péptido beta amiloide 1-42, comparando las poblaciones neuronales de la red septum hipocampo en un grupo de animales tratados con beta amiloide 1-42 y extracto metanólico de Lepidium meyenii.

Objetivo: Estudiar la capacidad neuroprotectora del extracto metanólico de Lepidium meyenii en un modelo de Alzheimer in vivo inducido por la administración de beta amiloide en las redes neuronales septum-hipocampo.

Procedimiento: Se utilizaron 24 ratas (Rattus norvegicus) machos de la cepa Sprague Dawley de 3 meses de edad con un peso aproximado de 250gr.La variedad de maca utilizada será la maca negra obtenida del departamento de Junín - Perú. Se realizó cirugía estereotáxica para inocular el péptido beta amiloide y extracto metanólico de Lepidium meyenii, para ello se utilizó el atlas Paxinos y Watson. Se utilizó anticuerpos que detecten microglías como Iba1(Abcam, EUA) y astrocitos GFAP (Abcam, EUA).

Resultados Preliminares: Inmunoreactividad de microglías (Iba1) en el área del Septum medial; 40x: En el grupo tratado con beta amiloide y Fracción acuosa del extracto metanólico de maca negra, se observa una disminución de la Inmunoreactividad de microglías en comparación con el grupo tratado solo con beta amiloide (1-42).

Conclusiones: ConrespectoalaInmunoreactividad de microglías, se observó un incremento de microglías activadas en el grupo de ratas tratadas con beta amiloide (1-42) en el área del septum medial en comparación con el grupo que fue tratado con beta amiloide y Fracción acuosa del extracto metanólico de maca negra. 


\section{LFP COMO MODELO DE EVALUACIÓN EN TRAUMATISMO CRANEOENCEFÁLICO EN UN MODELO ANIMAL.}

Gonzales, A., Minaya, V., Pacheco, L. F. y Aguilar, L. A. (Laboratorio de Neurociencia y Comportamiento. Universidad Peruana Cayetano Heredia).

Introducción: El traumatismo craneoencefálico es una de las principales causas en todo el mundo de causar lesiones con discapacidad permanente o la muerte, por año se llega a producir alrededor de 20 a 50 millones de traumatismo no mortales, siendo lesiones importantes para generar posterior discapacidad. Para su estudio se pueden subdividir en lesiones primarias y secundarias, cada una de ellas con características propias: fractura craneal, contusiones, laceraciones, lesión axonal o de la sustancia gris, del tronco encefálico, hemorragia intraventricular, lesiones intracraneales, hernias, isquemias, edema, hipoxia, entre otras. El daño generado puede evaluarse dependiendo de si la lesión es local o difusa y el progreso conlleva factores llamados también daño secundario. Debido a estos cambios tisulares, pueden desarrollarse, convulsiones, perdida de la autorregulación cerebral, inflamación, apoptosis o excitoxicidad.

Objetivo: Desarrollar un modelo de evaluación para traumatismo craneoencefálico y evaluar la respuesta inflamatoria producto del traumatismo por percusión lateral por fluido en un modelo animal.

Procedimiento: Para este trabajo se utilizarán 30 ratas macho de la cepa Sprague-Dawley con un peso promedio de 200-250gr. Distribuidas en grupos de traumatismo craneoencefálico leve, sham y control.Los animales se mantuvieron en el bioterio de la Universidad Peruana Cayetano Heredia, bajo condiciones de agua y comida ad libitum, temperatura controlada a $22^{\circ}$ y ciclos de luz / oscuridad cada 12 horas. Se realizó una craneotomía (Cirugía estereotáxica) +2 Lateral, -3.8 Antero-posterior a Bregma, 0 profundidad, luego una abertura de $3 \mathrm{~mm}$ de diámetro utilizando un micromotor y equipo de cirugía, se colocó un TIP plástico alrededor de la abertura. Luego del tiempo de recuperación se genera un traumatismo leve utilizando el equipo de percusión lateral por fluido o LFP.

Resultados Preliminares: En el momento se encuentra en la fase de desarrollo del modelo de
LFP, se ha desarrollado una variante del equipo de Percusión Lateral por Fluido (Virginia Commonwealth University), el equipo está compuesto por un péndulo que permite movilización a determinados ángulos generando determinada cantidad de fuerza, conectado a un cilindro inoxidable para la generación de presión y un sensor conectado con salida a un osciloscopio. La diagramación y diseño de los componentes del equipo han sido desarrollados mediante el software AUTOCAD. Actualmente en la fase de producción se desarrolla un sensor electrónico acondicionado para la trasformación a PSI y se realizan los trabajos de entrenamiento en el modelo animal.

ESTUDIO DEL APRENDIZAJE ESPACIAL Y LA ACTIVIDAD DE RITMOS THETA EN UN MODELO DE ALZHEIMER

Jhonatan-Astucuri, Luis-Pacheco, LuisAguilar. (Laboratorio de Neurociencia y Comportamiento, Universidad Peruana Cayetano Heredia)..

Introducción: La enfermedad de Alzheimer (EA) en un cerebro es progresivo y fatal, una de cada ocho personas mayores de 65 la padece. Se caracteriza por: aglomeraciones neurofibrilares producidas por la hiperfosforilación de la proteína TAU y; por el deposito excesivo de placas amiloides constituidas por la proteína $\beta$ amiloide. La actividad Neuronal puede ser fácilmente medible con equipos de registro EEG. Se puede registrar y analizar las alteraciones de los ritmos beta, theta beta y gamma en el córtex motor utilizando un equipo estereotáxico y electrodos implantables con un sistema de telemetría adjunto para realizar grabaciones.

Objetivo: Estudiar el aprendizaje espacial y la actividad eléctrica de ritmos theta en la Corteza Entorrinal en un modelo animal de Alzheimer inducido por $\beta$ amiloide.

Metodología: El estudio es prospectivo analítico, la población estaba constituida por ratas Sprague Dowley machos de tres meses de edad. Se utilizó el laberinto acuático de Morris para evaluar el deterioro del aprendizaje y memoria espacial. Se trabajo con un modelo de Alzheimer inducido por $\beta$-amiloide, en tres diferentes grupos de animales (experimental, sham y 
control). Se inoculo la proteína $\beta$ amiloide en zona de la corteza entorrinal (Anteroposterior: $-7.0 \mathrm{~mm}$, Lateral: + $5.4 \mathrm{~mm}$ y Vertical -8.0) ubicada gracias al atlas de Paxinos. Los registros electrofisiológicos se realizaron mediante cirugía estereotáxica; se realizó un implante agudo de electrodos cerebrales profundos en el septum medial. Finalmente se realizaron las grabaciones de los registros neuronales con el Software SciWorks con el plugin Experimenter.

Resultados: Respecto a la memoria espacial, se puede observar un aumento en la latencia de tiempo que demora el individuo al encontrar su objetivo. Los ritmos theta hipocampales disminuyeron en el septum medial.

Conclusiones: La EA deteriora significativamente el aprendizaje y memoria espacial al deteriorar la comunicación por medio de ritmos theta de las neuronas responsables de esta habilidad.

\section{POSTERS}

\section{ACTIVIDAD NEUROINMUNOMODULADORA DE LA Uncaria tomentosa SOBRE EL SISTEMA NERVIOSO CENTRAL, EN UN MODELO DE NEUROINFLAMACIÓN INDUCIDO POR LIPOPOLISACÁRIDOS (LPS) EN ANIMALES DE EXPERIMENTACIÓN}

Vásquez, R., Andrade, R., Aguilar, L. A. (Laboratorio de Neurociencia y Comportamiento, Universidad Peruana Cayetano Heredia).

Aguilar, L. (Laboratorio de Neurociencia y Comportamiento, Universidad Peruana Cayetano Heredia Laboratorio de Inmunología, Universidad Peruana Cayetano Heredia).

Introducción: Las glías son células inmunes residentes en el sistema nervioso central (SNC) que son activadas en respuesta a un daño cerebral. Al activarse, las glías liberan varios factores neurotróficos los cuales van a favorecer la supervivencia neuronal, pero también inducen factores neurotóxicos que incluyen al Óxido Nítrico (ON) y sustancias normales de defensa que secretadas en exceso pueden ser también neurotóxicas como citoquinas pro-inflamatorias IL$1 \beta$, IL-6 o TNF- $\alpha$. Mientras que la activación glial es necesaria y crítica para la defensa innata, su sobre activación es neurotóxica. Además, la activación glial juega un rol importante en la progresión de enfermedades neurodegenerativas como el Alzheimer o el Parkinson.

Objetivos y Procedimiento: En el presente estudio se evaluó la acción neuroinmunomoduladora de la Uncaria tomentosa en la inhibición de activación de astrocitos, a nivel del sistema nervioso central (SNC). Se utilizó ratas Sprague-Dawley $(240-260 \mathrm{gr})$, las cuales fueron mantenidas en condiciones de laboratorio con libre acceso a comida y agua, a una temperatura ambiente de $21^{\circ} \mathrm{C}$, y ciclos a 12 horas de ciclo de luz diurna (luz a las 7:00 h). Los animales fueron tratados antes de la cirugía estereotáxica con Uncaria tomentosa $(500 \mathrm{mg} / \mathrm{kg}$ y $100 \mathrm{mg} / \mathrm{kg}$ ) administrada vía oral con sondas orogástricas $\mathrm{N}^{\circ} 18$, durante quince días. Luego se inyectó $2,5 \mu \mathrm{L}$ de LPS en cada ventrículo lateral (bregma: - 0.9mm; Lateral: $\pm 1.8 \mathrm{~mm}$; Vertical: $3.8 \mathrm{~mm}$ ). Los animales fueron sacrificados luego de 16 horas de aplicado el LPS para la evaluación inmunohistológica a nivel del Hipocampo.

Resultados: En el área hipocampal CA1, el promedio de astrocitos activados fue de 26.1 y 54.3, en los grupos tratados con $500 \mathrm{mg} / \mathrm{kg}$ y $100 \mathrm{mg} / \mathrm{kg}$, respectivamente; los cuales menores en comparación al grupo control que tuvo 89.3 astrocitos activados $(\mathrm{p}<0.05)$. En el área CA2, el promedio de astrocitos activados fue de 23.1 y 41.67 , en los grupos tratados de $500 \mathrm{mg} / \mathrm{kg}$ y $100 \mathrm{mg} / \mathrm{kg}$, respectivamente; los cuales fueron estadísticamente menores al grupo control que tuvo 74.5 astrocitos activados $(\mathrm{p}<0.05)$.

Conclusión: La Uncaria tomentosa (uña de gato), presentó un efecto neuroprotector en el hipocampo de animales experimentales, siendo necesario evaluar los mecanismos moleculares que ejercen dicho efecto neuroprotector.

\section{IMPLEMENTACIÓN DE UN KIT MECATRÓNICO DE DESARROLLO ACADÉMICO PARA LA SINTONIZACIÓN DE SISTEMAS DE CONTROL ESTADÍSTICOS}

Calluco Espinoza, W. (Promaitec Ingenieros E.I.R.L.).

Introducción: Adquirir conocimientos para la sintonización de los parámetros características de 
un sistema de control PI, PID y Adaptativo de los mecanismos sencillos y complejos de las maquinas industriales son de relevancia, para sistemas de operación continua ya que esto implica minimizar el desgaste y las fallas. Realizar simulaciones en un kit de desarrollo del sistema de control en un prototipo nos da una estimación de los diferentes efectos producidos de tal manera que se pueda tomar decisiones correctivas, preventivas y predictivas en los sistemas mecánicos, que casi siempre están expuestos a perturbaciones dinámicas.

Objetivos: En el presente proyecto que se encuentra en actual desarrollo, se busca la formación de carácter intuitivo y cualitativo de los estudiantes de universidades, en conocimientos de sistemas de control, así como la comprensión intrínseca de los conocimientos de programación en sistemas con retroalimentación (feedback).

Procedimiento: Para esto se realizaron simulaciones del sistema en simulink (Matlab), y se aplicaron teorías de ingeniería de control utilizando el algoritmo de control adaptativo por planificación de ganancias, así como el modelamiento cinemático y dinámico de los sistemas aplicando algoritmos computacionales. Para la implementación utilizamos módulos de desarrollo integrados por microcontroladores y sensores de bajo consumo de energía.

Resultados: Lo resaltante del proyecto es la comprensión del conocimiento adquirido que tiene un alcance multidisciplinario ya que no solo interactúan estudiantes de las carreras de ingeniería sino también de otras ciencias, ya que para lograr la sintonización del sistema es en base al ensayo de prueba y error, pudiéndose contrastar los conocimientos de la teoría con la experimentación. El kit de desarrollo es escalable razón por la cual su prototipado puede ser extendido a mecanismos más complejos. Con este kit de desarrollo se puede sistematizar y documentar los resultados obtenidos en los ensayos para poder generar una base de datos de sencilla transferencia.

Conclusiones: Se concluye que la implementación del kit mecatrónico permite la comprensión interactiva e intuitiva de los sistemas de control con realimentación, desarrollando en el usuario patrones de sintonización.
IN SILICO ANALYSIS OF CELL NUCLEI ON DIFFERENT CELLULAR STRESS MODELS

Hernández, D. ${ }^{1}$, Heman, P., Rosas, C. ${ }^{2}$, Barrientos, A. ${ }^{3}$, Pensado, P., Sandoval, $C^{3}$, Nadella, R. ${ }^{4}$, , Zavala, $L .{ }^{5}$, Villanueva, A. ${ }^{6}$

1. CONACYT-Instituto de Neuroetologia, Universidad Veracruzana -México.

2. Posgrado en Neuroetologia Universidad Veracruzana

3. Facultad de Química Farmacéutica Biológica Universidad Veracruzana. México.

4. Department of Biotechnology, Sri PadmavatiMahi laVisvavidyalayam, Tirupati; CONACyT-Instituto de Neuroetologia - India.

5. Centro de Investigación Biomédica del Noreste - México.

6. Facultad de Medicina. Universidad Autonoma de Nuevo León -México.

Introduction: The process of induction of cellular stress by diverse stimuli is accompanied by cellular processes such as inflammation, degeneration and cell death. Although there are various immunostaining techniques for diagnostic studies using haematoxylineosin, the analysis of neuronal nuclear patterns (or of somatic cells) has been neglected.

Objective: To determine nuclear morphometric patterns in neurons and in somatic cells on diverse stress models.

Procedure: In this work, cellular stress was induced by four different approaches: 1) intraperitoneal administration of lipopolysaccharide, an immune response activator, 2) intranigral injection of Staurosporine, an apoptosis enhancer, 3) partial hepatectomy in rats and 4) cytotoxicity assay in $\mathrm{SH}$ SY5Y cell lines. The tissue sections $(5-10 \mu \mathrm{m})$ or cells preparations (monolayer) were immunostained with Hoechst 33342 (nuclear marker), CD11b/cOX-42 (microglial marker), tyrosine hydroxylase (dopaminergic phenotype marker) and caspase-3 (apoptosis marker). To evaluate shape, size, number and hyper/hypochromic features, we used ImageJ software.

Results: During inflammation, degeneration and cell death processes, particulars regarding the nuclear patterns were distinguished.

Conclusion: Regardless of the stressor stimulus, the analysis of the nuclear patterns contributes to the histopathological diagnosis. 


\section{INFLUENCIA DE LA MASTICACIÓN DEFICIENTE SOBRE LA MEMORIA Y EL APRENDIZAJE ESPACIAL}

Aguirre Siancas, E. (Universidad Nacional Mayor de San Marcos, Programa Doctoral en Neurociencias, Lima, Perú. Universidad Científica del Sur, Escuela de Estomatología, Lima, Perú).

Introducción: La memoria y el aprendizaje son funciones cardinales en todos los seres vivos. En los seres humanos, el deterioro de dichas funciones tiene un impacto devastador en las personas, las familias y la sociedad en su conjunto. Muchas causas que aún no son tan claras explican este deterioro, dentro de ellas está la masticación deficiente.

Objetivo: Determinar la influencia de la masticación deficiente sobre la memoria y el aprendizaje espacial en ratones albinos machos y adultos de la cepa $\mathrm{Balb} / \mathrm{c} / \mathrm{CNPB}$.

Procedimiento: Estudio experimental realizado en 20 ratones albinos machos de 5 meses de edad de la cepa Balb/c/CNPB. Los animales se dividieron en 2 grupos iguales, el grupo masticación normal fue mantenido con alimentación en granos, la misma que recibieron los 20 ratones desde el destete; el grupo masticación deficiente fue alimento con los mismos granos, pero pulverizados. Se evaluaron a los animales al séptimo mes de vida en la fase de adquisición y luego en la fase de recuperación de memoria y aprendizaje espacial en el laberinto acuático de Morris. Al encontrarse normalidad en la distribución de los datos y homocedasticidad se empleó la prueba t de student para muestras independientes. El nivel de significancia considerado fue de 0,05 .

Resultados: Se encontraron diferencias significativas en el primer día $(p=0,035)$ de la fase de adquisición a favor del grupo masticación normal. En el resto de días de dicha fase no hubo diferencias estadísticas entre ambos grupos $(p>0,05)$, aunque siempre el grupo masticación normal tuvo mejor performance vs el grupo masticación deficiente. En la fase de recuperación no hubo diferencias.

Conclusión: Estos resultados sugieren que la masticación deficiente influye negativamente en la formación de memoria y aprendizaje espacial en ratones albinos machos y adultos de la cepa Balb/c/ CNPB.

\section{PERCEPCIÓN DE CUIDADORES Y PROFESIONALES EN PSICOLOGÍA, FRENTE AL USO Y EFECTIVIDAD DE TERAPIAS BASADAS EN EL MÉTODO ABA (APPLIED BEHAVIOR ANALYSIS) PARA EL TRATAMIENTO DE PERSONAS CON AUTISMO.}

Bejarano, A.,León, M., Linares,S. (Corporación Universitaria Iberoamericana, Bogotá Colombia, Facultad de Ciencias Humanas y Sociales).

Introducción: El enfoque cognitivo conductual (del cual se deriva el método ABA) se caracteriza por ser sensible al contexto y a las funciones de los fenómenos psicológicos con el fin de buscar repertorios amplios, flexibles y efectivos que permita afrontar las dificultades, en lugar de buscar su eliminación. La evidencia a favor que se ha acumulado durante los últimos años en relación con este tipo de método (ABA), ha permitido posicionarse como uno de los más efectivos en el tratamiento de distintos trastornos, especialmente en lo que tiene que ver con el Trastorno de Espectro Autista.

A partir de lo anterior, se busca indagar en las percepciones que tienen profesionales en psicología y cuidadores de personas con trastorno del espectro autista "TEA" frente al uso y efectividad de los tratamientos basados en el método ABA (AppliedBe haviorAnalysis),

En Colombia y todo el mundo el diagnóstico del trastorno del espectro autista "TEA", ha ganado mayor visibilidad en los últimos años, Según el centro para el control y prevención de enfermedades de EEUU (CDC), 2009 "la tasa mundial de Trastorno del Espectro Autista (TEA o autismo) es de 1 de cada 110, hasta el momento Colombia no cuenta con cifras oficiales que establezcan la prevalencia en el país de este trastorno" (Ministerio de Salud y Protección Social., 2013).

Objetivo: Comparar las percepciones entre profesionales en psicología y cuidadores de personas con autismo, frente al uso y efectividad del método $\mathrm{ABA}$ en los procesos terapéuticos e interventivos dirigidos a la población con TEA.

Según lo explicado por Sampieri, Fernández y Baptista (2010) esta investigación es de enfoque mixto, con un diseño de ejecución concurrente, es decir, 
ambos métodos se aplican de manera simultánea; ni el análisis de los datos cuantitativos, ni cualitativos se construyen sobre la base del otro análisis, con lo que se pretende integrar las inferencias y conclusiones de los datos realizados de manera independiente.

El grupo poblacional utilizado para esta investigación serán voluntarios profesionales (3) y cuidadores (3) de personas con TEA, que se encuentren bajo el método $\mathrm{ABA}$ hace más de seis meses

A partir de allí, se espera obtener información con la cual se pueda desarrollar un análisis comparativo y obtener una visión sobre la situación actual que viven las personas con autismo en relación a los procesos de intervención y acompañamiento a los que puede acceder.

\section{MARCADORES GENÉTICOS PARA TOC (TRASTORNO OBSESIVO COMPULSIVO) Y TDAH (TRASTORNO POR DÉFICIT DE ATENCIÓN E HIPERACTIVIDAD) EN POBLACIÓN COLOMBIANA}

Pinto, M., Polanco, A., Ávila, J., Arboleda, H. y

Vásquez, R. (Universidad Nacional de Colombia - Colombia).

Introducción: En el grupo de Neurodesarrollo de trastornos de comportamiento de la Universidad Nacional se han realizado en los últimos años, una serie de estudios con el fin de dar cuenta de los marcadores biológicos que se pueden asociar a problemas de comportamiento en la población infantil colombiana.

Objetivos: Analizar la relación de polimorfismos con el riesgo de presentar Trastorno por déficit de atención e hiperactividad (TDAH) y el trastorno obsesivo compulsivo (TOC).

Procedimiento: Con respecto a TDAH se ha buscado la presencia de polimorfismos del gen DGKH (Diacilglicerol Quinasa Eta), DAT1 VNTR 3’ UTR y DRD4 VNTR Exón III, para el caso de TOC

Resultados: Para el caso de TDAH se encontró una asociación significativa del SNP rs1170169 como protector, se obtuvo un p de 0.01097y un OR de 0,39 . Y una asociación de riesgo del SNP 9525580 para el trastorno con un p de 0.01453 y un OR 2, 5. DAT1 VNTR 3' UTR y DRD4 VNTR Exón III, el genotipo homocigoto de 10 repeticiones (10/10) de DAT1, aparece con mayor frecuencia en los dos grupos (Casos: 0,85; Control: 0,70), manifestando un OR: 2,5 (IC 95\%: 0,684- 9,133; p: 0,158). Para el polimorfismo DRD4, el genotipo homocigoto de 4 repeticiones (4/4) es el de mayor aparición, aunque en los casos, comparte la frecuencia $(0,38)$ con el genotipo $4 / 7$, que presenta el OR más elevado $(2,5$ IC 95\%: 0,827- 7,560 p: 0,1). En el caso de TOC los análisis a través de 6 polimorfismos genéticos; a saber, COMT, MAO-A, HTTLPR, HT2A, SLC1A1 y DRD4, los resultados mostraron relación entre los polimorfismos y funcionamiento neuropsicológico, pero no hay diferencias entre pacientes y controles.

Conclusiones: Para TDAH se confirma la asociación del trastorno con polimorfismos reportados en la literatura, para $\mathrm{TOCl}$ a falta de asociación con algunos polimorfismos puede estar relacionada con el tamaño de la muestra. Actualmente el grupo trabaja en un proyecto en el que se tendrán en cuenta 10 polimorfismos que por antecedentes investigativos ha estado asociados a trastornos psiquiátricos y a neurodesarrollo, y los promotores de los genes DLGAP1 y BTBD3.

\section{ANÁLISIS NEUROPSICOLÓGICO DE NIÑOS Y JÓVENES CON TRASTORNOS DE COMPORTAMIENTO}

Pinto, M., Polanco, A., Ávila, J., Arboleda, H. y

Vásquez, R. (Universidad Nacional de Colombia - Colombia).

Introducción: Los trastornos del neurodesarrollo son clínicamente heterogéneos incluyendo aquí manifestaciones en las diferentes habilidades cognoscitivas, constituyéndose estas en formas de aproximarse a modelos funcionales y a partir de esto a posibles medidas de intervención. Desde las investigaciones realizadas con Trastornos de comportamiento, dentro del Grupo de Neurociencias de la UNAL, se han identificado hallazgos relacionados principalmente con el funcionamiento de los sistemas Atencional, Velocidad de procesamiento, memoria de trabajo y regulación por parte de la Función ejecutiva, en trastornos como el TDAH y el TOC.

Objetivos: Caracterizar el funcionamiento cognitivo de niños y jóvenes con trastornos de comportamiento. 
Procedimiento: Para los grupos de TDAH se usaron subcomponentes del WISC y para el estudio de TOC se hizo una evaluación de funciones ejecutivas a partir del test WCST, TMT, Stroop y Torre de Londres.

Resultados: En el TDAH, se identifica que quienes manifiestan este cuadro difieren significativamente con un nivel de significancia p: 0,05 , en el rendimiento tanto Intelectual global, como en índices específicos -CI total (p: 0,00) Índice de comprensión verbal (p: 0,00), Claves (p: 0,00), ENI Cancelación de dibujos (p: 0,018$)$ y Stroop Interferencia (p: 0,00)- del grupo control. Dentro de la caracterización neuropsicológica, al diferenciar el grupo de TDAH con trastorno de ansiedad (TDAH+TA) mostró un rendimiento cognoscitivo menor en general, mientras el grupo de TDAH sin comorbilidad evidenció una alteración en inhibición como parte de la función ejecutiva. Al hacer evaluaciones relacionadas con el TOC, se encontró que los Pacientes mostraron mejor desempeño en algunos subpuntajes del WCST en comparación con los controles. En las demás pruebas no se alcanzaron niveles de significancia para establecer diferencias entre los pacientes y controles.

Conclusiones: Se propone un nuevo tipo de análisis de asociación al vincular características Genéticas y Neuropsicológicas, independientemente de la manifestación de un trastorno específico: Por ejemplo; se identifica al hacer análisis de riesgos que quienes presentan el alelo de 7 repeticiones del gen del receptor de Dopamina DRD4 (alelo de riesgo para el TDAH); tienen un riesgo mayor de presentar un bajo rendimiento (menor a 1DT) en las pruebas relacionadas con velocidad de procesamiento y atención selectiva dentro de un límite de tiempo, lo que afecta el sostenimiento atencional -Velocidad de procesamiento y búsqueda de símbolos. Esta propuesta de análisis representa una forma nueva de aproximación que brinda utilidad para la comprensión del riesgo y la formulación de modelos integrales y explicativos.

VARIABLES CLIINICAS Y AMBIENTALES DEL NEURODESARROLLO EN EL TRASTORNO DEL COMPORTAMIENTO

Pinto, M., Polanco, A., Ávila, J., Arboleda, H.y Vásquez, R. (Universidad Nacional de Colombia - Colombia).
Introducción: Parte de los modelos explicativos, para comprender los distintos trastornos del comportamiento, es identificar posibles aspectos relacionados como factores ambientales, socioculturales así como los eventos relevantes en el desarrollo como los son los antecedentes pre y perinatales y siendo aún mucho más específicos la interrelación existente con el mismo ambiente a través de por ejemplo los patrones de crianza, cuidados durante el desarrollo e identificación de antecedentes médicos dentro de la misma familia.

Objetivos: Identificar algunos de los principales factores de riesgo y predisponentes familiares y ambientales relacionados con la presencia de trastornos de comportamiento en niños y jóvenes colombianos.

Procedimiento: Se ha establecido hacer un estudio de asociación entre estos factores medioambientales, socioculturales y del neurodesarrollo y su posible asociación con el trastorno de comportamiento y las variaciones entre ellas; para ello se diseñó un instrumento (cuestionario) para identificar los antecedentes a través de una entrevista, se construyó una base de datos para la organización de los datos y favorecer su análisis y se aplicó un protocolo de evaluación clínica dirigido por un grupo psiquiatría infantil del Hospital de la Misericordia para corroborar el trastorno de comportamiento y como tal hacer el perfilamiento de cada uno de los casos y así ver diagnóstico diferencial entre ellos.

Resultados: Se encuentran relaciones entre la presencia del trastorno y aspectos relacionados con la crianza, aspectos asociados a la gestación y el tipo de alimentación durante los primeros meses.

Conclusiones: El grupo de Neurociencias de la Universidad Nacional de Colombia, ha evidenciado dentro ellas el que algunos antecedentes en el neurodesarrollo, factores ambientales, culturales, etc. han tenido una alta asociación con el Trastorno por Déficit de Atención e Hiperactividad (TDAH) en sus variaciones con y sin ansiedad. Sin embargo, lo fundamental es aumentar muestras y ser más minucioso en estos factores medio ambientales y generar posibles marcadores epigenéticos. 


\section{REVISIÓN SISTEMÁTICA DE LOS ESTUDIOS EN TORNO A LA RELACIÓN ENTRE ANTICONCEPTIVOS HORMONALES, ESTRÓGENOS Y DESEMPEÑO EN TAREAS DE MEMORIA.}

Buritica, E. \& Cruz, D. (Estudiantes de la Corporación Universitaria Iberoamericana. Colombia).

Introducción: La revisión sistemática es un estudio investigativo retrospectivo en el cual se combinan estudios que se examinan a partir de una misma pregunta problema buscando identificar datos significativos que le permitan al lector tener una puesta al día sobre conceptos útiles en áreas de constante evolución; nuestra área de interés ha mostrado algunos resultados en el campo de la investigación de procesos cognitivos y hormonas.

Objetivo: Identificar a través de una revisión sistemática en la base Web of Science, las tendencias investigativas en torno al estudio de la relación e influencia entre hormonas sexuales (estrógeno), utilización de anticonceptivos hormonales y desempeño en tareas de memoria

Procedimiento: Nuestro modelo metodológico está basado en la declaración PRISMA para la elaboración de revisiones sistemáticas en la cual se describe minuciosamente en 27 ítems el paso a paso para la correcta construcción de meta-análisis y revisiones sistemáticas. De acuerdo a estos lineamientos, en primer lugar, hemos elaborado diagramas de flujo y categorización de la información como base de nuestra revisión, tal cual lo sugiere la declaración PRISMA.

Resultados Parciales: En el momento se cuenta con la clasificación de los artículos de acuerdo a las categorías y diagramas que se establecen inicialmente (memoria, ciclo menstrual, anticonceptivos, hormonas) donde, por ejemplo, ya se puede ver que 16 investigaciones centran su interés en lo que sucede a nivel de memoria en la fase lútea del ciclo menstrual. Se espera consolidar la información para entregar datos más detallados según las categorías que hemos establecido.

\section{TRANSFERENCIA DE FUNCIONES SINTÁCTICAS Y EL PROCESAMIENTO DE CAMBIO DE CÓDIGO LINGÜÍSTICO EN MONOLINGÜES Y BILINGÜES}

Astucuri, J. ${ }^{1}$, Espinoza, V. ${ }^{2}$, Achanccaray, D. ${ }^{3}$,

Pirca, J. ${ }^{4}$ y Aguilar, L. ${ }^{1}$

1. Laboratorio de Neurociencia y Comportamiento, Universidad Peruana Cayetano Heredia

2. Facultad de Ingeniería Económica, Universidad Nacional de Ingeniería.

3. Grupo de Investigación en Robótica Aplicada y Biomecánica, Pontificia Universidad Católica del Perú.

4. Grupo de Investigación en Robótica Aplicada y Biomecánica, Pontificia Universidad Católica del Perú.

Introducción: El fenómeno de lenguas de contacto ha buscado diferenciar el concepto de préstamo y cambio de código. Si bien el préstamo es más frecuente, también el cambio de código en calidad discursiva habitual, por lo que se utilizó gramática artificial para evaluar la capacidad de transferencia de los sujetos, mediante la asimilación de reglas y aplicación a patrones de letras.

Objetivo: Determinar la relación entre la transferencia de funciones sintácticas y el procesamiento de cabios de códigos lingüístico en monolingües y bilingües.

Procedimiento: El tipo de estudio es analítico debido a que se realizó un análisis según el registro eléctrico durante las actividades de gramática artificial en las que se evaluó los N400. Consiste en la evaluación de secuencias gramaticales y no gramaticales, comparando los resultados de 18 participantes voluntarios (9 participantes adultos pertenecientes al grupo monolingüe y 9 participantes adultos bilingües con certificación internacional de inglés según el marco europeo - FCE). El procedimiento se llevó a cabo en una sesión de entrenamiento de gramática artificial en la que los participantes debían memorizar y transcribir las secuencias "gramaticales", para lo que se usó la gramática de estado finito de Meulemans. La regla combinatoria utilizó 6 caracteres distintos, uno de entrada y dos de salida. Los sujetos realizaron la tarea de gramática artificial en forma individual.

Resultados Parciales: Con este entrenamiento se observó un mejor desempeño en la fase de transferencia como paradigma experimental para el estudio de los procesos de adquisición del lenguaje de los bilingües. 
Conclusiones: Los bilingües presentan mayores facilidades que los monolingües al formar nuevas estructuras esto puede deberse a que cuentan con un sistema de leyes más complejo, el cual les permite liberar su potencial lingüístico en menor tiempo.

\section{RESPUESTA AL ESTRÉS OXIDATIVO EN UN MODELO EN RATAS CON NEUROCISTICERCOSIS}

D. G. Dávila ${ }^{1}$, R. P. Carmen ${ }^{1}$, R. H. Gilman ${ }^{2}$, Y. Cauna1, M.Criollo ${ }^{1}$, G. Castillo1,E.G.Bernal ${ }^{1}$, A. D. Delgado ${ }^{1}$, R. H. Céliz, M. R. Verastegui ${ }^{1}$

1. Laboratorio de Investigación de Enfermedades Infecciosas - LID. Universidad Peruana Cayetano Heredia, Lima, Peru.

2. Johns Hopkins University, Baltimore, MD, UnitedStates

Introducción: La Neurocisticercosis (NCC) afecta al Sistema Nervioso Central (SNC) y es causada por la infección del metacéstode de Taenia solium. En NCC, las lesiones cerebrales son dependiente de la respuesta inmune-inflamatoria. Se ha reportado que en enfermedades neurológicas la vía de NO sintasa (NOS) se ve afectada. La alta actividad de la vía NO sintasa inducidos por los factores inflamatorios contribuyen en el estrés oxidativo y por lo tanto cumplen un rol importante en la severidad de la enfermedad. Es conocido que, en diversas enfermedades como la encefalopatía hepática, enfermedades cardiovasculares existe la alteración del metabolismo de $\mathrm{NO}$, sin embargo, esta información permanece pobremente entendida en NCC.

Objetivo: Determinar el cambio en el nivel de expresión de los siguientes genes Nos1, Nos2, Nos3, Arg1, Arg2 y Sod1 por el ensayo de PCR de transcriptasa inversa en tiempo real (RT2- PCR) en ratas para NCC ratas Holtzman de entre 8 -10 días de nacidas.

Procedimiento: Se inocularon intracranealmente oncósferas activadas de Taenia solium. Después de 4 meses las ratas infectadas desarrollan quistes en el tejido cerebral. Los animales fueron sacrificadas y el tejido alrededor del quiste fueron colectados $(n=11)$ y controles $(n=12)$. Las muestras fueron preservadas en RNA later. Luego se extrajo el RNA, se sintetizó el cDNA usando un kit RT, y finalmente se procedió a la cuantificación relativo por el ensayo de PCR de transcriptasa inversa (RT-PCR) en tiempo real.

Resultados: Los resultados del ensayo de transcriptasa inversa en tiempo real (RT2-PCR) muestran que la expresión de mRNA de Nos2 y Arg1 fue significativamente mayor en muestras infectados comparado con los controles $(\mathrm{p}<0.007$ y $\mathrm{p}<0.00)$, mientras que la expresión mRNA de Nos1, Nos3, Arg2 y Sod1 fueron comparables.

Conclusión: En el presente trabajo, la expresión de Nos2 y Arg1 fueron significativamente mayor en tejidos alrededor del quiste sugiriendo el rol de estas moléculas en la patogénesis de la enfermedad de NCC.

\section{APLICACIÓN DEL SISTEMA DE E L E C T R O E N C E F A L O G R A M A TELEMÉTRICO EN RATAS CON NEUROCISTICERCOSIS}

A. D. Delgado ${ }^{1}$, R. P. Carmen Orozco ${ }^{1}$, R. H. Gilman $^{2}$,F.Ancajima ${ }^{1}$,R.H.Celíz ${ }^{1}$,D.G.Dávila Villacorta $^{1}$, E. Bernal ${ }^{1}$, L. E. Baquedano ${ }^{1}$, M. R. Verasteguii ${ }^{1}$.

1. Laboratorio de Investigación de Enfermedades Infecciosas - LID. Universidad Peruana Cayetano Heredia, Lima, Perú.

2. Johns Hopkins University, Baltimore, MD, United States

Introducción: La Neurocisticercosis (NCC), es la zoonosis parasitaria generada por la larva del metacéstode Taenia solium, que infecta el sistema nervioso central. Es la principal causa de epilepsia tardía en adultos de zonas endémicas y se estima que entre un 70 a $90 \%$ de personas con NCC llegan a desarrollar epilepsia. La epileptogénesis de la NCC aún no ha podido ser estudiada, ya que no se cuenta con modelos animales que presenten una semiología semejante a la humana que desarrolle cuadros de convulsiones espontaneas.

Objetivo: Nuestro objetivo es determinar si existen cambios en el registro de electroencefalograma telemétrico (tEEG) asociados con convulsiones espontaneas en ratas con neurocisticercosis. 
Procedimiento: Se utilizó ratas Holtzman macho entre 12 y 15 días de nacidas, adquiridas en el bioterio de la Universidad Peruana Cayetano Heredia. Se realizó la infección intracraneal con 360 oncósferas de Tenia solium activadas, inoculadas a nivel del bregma del cráneo. Se seleccionaron ratas infectadas $(\mathrm{n}=8)$ y ratas no infectadas $(\mathrm{n}=3)$, a las cuales se les implantaron dos electrodos corticales; el registro de tEEG se realizó 24/7 evaluando la actividad cerebral durante 5 semanas.

Resultados: En el grupo de ratas infectadas con $\mathrm{NCC}(\mathrm{n}=8)$, se observaron alteraciones en el registro de tEEG en el 50\% $(n=4)$, identificadas como descargas generalizadas tipo punta, con una duración media de 50 segundos por convulsión; en comparación con el grupo control, en donde no se observaron alteraciones en la actividad base que pudieran ser identificadas como convulsiones espontaneas.

Conclusión: En conclusión, el registro de Electroencefalograma telemétrico nos permite identificar descargas paroxísticas generalizadas tipo punta, las cuales son asociadas con convulsiones espontaneas.

\section{ESTUDIO SOBRE HÁBITOS DE SUEÑO Y SOMNOLENCIA DIURNA EN MUJERES UNIVERSITARIAS DE UNA UNIVERSIDAD PERUANA}

Cárdenas, A.l. ${ }^{1}$, Castro, M.j. ${ }^{1}$, Gonzales, A. ${ }^{1}$, Cisneros, R.1, ${ }^{2}$, León, R. ${ }^{1,2}$, Malpartida, C. ${ }^{\text {, }}$ Muñoz, A. ${ }^{3}$, Loayza L ${ }^{3}$, Aguilar, L. $A^{3}$.

1. Laboratorio de Neurociencia y Comportamiento, Universidad Peruana Cayetano Heredia. Lima, Perú

2. Universidad Femenina del Sagrado Corazón. Lima, Perú.

3. Facultad de Ciencias de la Salud. Universidad San Ignacio de Loyola. Perú.

Introducción: El sueño es una actividad neurofisiológica importante para la calidad de vida de las personas. Para los estudiantes llevar buenos hábitos e higiene de sueño son importantes para la mejora de las actividades cognitivas como el aprendizaje y memoria. Sin embargo, en la vida universitaria la mayoría realizan actividades previas a dormir (p. ej., uso de tecnologías y consumo de sustancias psicoactivas) que influyen de manera negativa en la calidad de sueño y en la somnolencia diurna, afectando así los procesos cognitivos.

Objetivo: Valorar la calidad de sueño en estudiantes universitarias mujeres de una universidad peruana.

Procedimiento: Se estudiaron a150 mujeres universitarias, de entre 16 y 33 años $(M=19,5$; DT= $02,59)$ donde les aplicó el "Cuestionario sobre hábitos de sueño" y la "Escala modificada de somnolencia de Epworth". Se analizaron los datos por medio de un programa estadístico - Rho de Spearman.

Resultados: Los resultados muestran que duermen una media de 06 horas y media. Encontramos que el $7,4 \%$ de las alumnas presentan hábitos de sueño malos, el $65.3 \%$ presentan hábitos de sueño regular, el $27.3 \%$ presentaron hábitos de sueño apropiados, mientras que ninguna presentó hábitos de sueño excelentes. Por último, dentro de los rangos de la escala de EPWORTH, encontramos que el $6.7 \%$ de las alumnas contaban con somnolencia leve y el mismo porcentaje se halló para somnolencia excesiva, mientras que, para el rango de somnolencia moderada, se encontró un $86,6 \%$.

Conclusiones: Existe una correlación inversa mínima entre los hábitos de sueño y la somnolencia donde inadecuados hábitos de sueño provocan mayor somnolencia. Las prácticas inadecuadas de sueño y su relación con la somnolencia nos llevan a recomendar el desarrollo de un programa de promoción de buenas prácticas de higiene de sueño en estudiantes universitarias, teniendo en cuenta que hay estudios que señalan que el sueño y la somnolencia inciden en el rendimiento académico.

\section{RESEARCH ON ABNORMAL EXPRESSION OF NA+ $/ \mathrm{K}+$ ATPASE A-SUBUNITS IN HIPPOCAMPUS OF A BASIC MODEL OF EPILEPTICS}

V. Minaya ${ }^{2}$., V.r Funck ${ }^{3}$, R. Treviño1., J. Astucuri $^{2}$., A. Gonzales ${ }^{2}$., O. Nuñez ${ }^{2}$, R.e. Andrade $^{2}$,, R.a. Cisneros ${ }^{2}$, Er. GarridoSanabria ${ }^{1}$, L.f.Pacheco ${ }^{1,2}$, M.Perez $^{4}$, A Muñoz $^{4}$, L. Loayza ${ }^{4}$ L. Aguilar ${ }^{4}$

1. Department of Health and Biomedical Sciences, the University of Texas at Rio Grande Valley, Brownsville, Texas, United States of America. 
2. Department of Sciences and Philosophy, Alberto Cazorla Talleri, the Universidad Peruana Cayetano Heredia, San Martin de Porres, Lima, Peru.

3. Graduate Program in Pharmacology, Federal University of Santa Maria, Santa Maria Brazil.

4. Faculty of Science of Health. University "San Ignacio de Loyola”. Perú

Background: Temporal lobe epilepsy is a condition characterized by frequent seizures occurring over time in one or both temporal lobes of the brain. Symptoms may be as mild as simple partial seizures, or as severe as a tonic-clonic seizure "Grand Mal seizure". The hippocampus has been shown to be involved in these types of seizures, and thus has been examined in how the expression of genes and proteins can possibly explain seizures in an individual that suffers disruptions in the normal balance of excitation and inhibition. $\mathrm{Na}+$ $\mathrm{K}+$ pump or sodium, potassium ATPase, is a crucial protein responsible for electrochemical gradient across the cell membrane. Major disruptions of $\mathrm{Na}+\mathrm{K}+$ pump functions can cause severe alterations in the neuronal role by controlling the activity of ion channels in the release of neurotransmitters and hormones through neuronal excitability, as well as synaptic plasticity and gene transcription.

Objective: In this study we are investigating whether the expression of the different ATPase isoforms are affected during epileptogenesis (acute period: $24 \mathrm{~h}$, a silent period: 10 days and the chronic period: 1 month and $>2$ months) following pilocarpine-induced status epilepticus.

Methods: For this purpose, we use ATPase activity, western blot assays and comparative analysis of gene expression using TaqMan-based probes and deltadelta cycle threshold (DDCT) methods of quantitative real-time polymerase chain reaction (qPCR) technique in samples obtained from age-matched control and epileptic rat.

Resultados: Abnormal expressions of sodium, potassium ATPase a3 isoform may contribute hyperexcitability of chronic epileptic rats. A marked up-regulation of a3 ATPase correspondingly, a $160 \%$ increase of protein levels was detected in the hippocampus of silent period 10days and chronic epileptic rats. Real-time quantitative PCR analysis of gene expression revealed that a significant $150 \%$ increase of transcripts for a3 ATPase occurred after 10days of pilocarpine-induced status epilepticus and persisted during the chronic phase of the model.

Conclusions: These data indicate a increase expression of a3 ATPase at protein and transcript levels in the epileptic hippocampus. Up-regulation of a3 ATPase in hippocampus may contribute to enhanced presynaptic excitability leading to recurrent seizures in the pilocarpine model of temporal lobe epilepsy.

\section{STUDY OF THE NEUROIMMUNOMO DULATION OF Uncaria tomentosa IN THE CENTRAL NERVOUS SYSTEM, A BASIC MODEL OF NEUROINFLAMMATION}

Gonzales, $\mathbf{A}^{\mathbf{1}}$, Cisneros, $\mathbf{R}^{\mathbf{1}}$, Andrade, $\mathbf{R} .{ }^{1}$, Yseki, D. ${ }^{1}$, Caballero, D. ${ }^{1}$, Minaya, $\mathbf{V}^{1}$, Gomez, J. ${ }^{2}$, Zavaleta J. ${ }^{2}$, Muñoz A. ${ }^{2}$, Aguilar, La. ${ }^{2}$

1. Laboratory of Neuroscience and Behavior. Cayetano Heredia University, Peru .

2. Faculty of Science of Health. University "San Ignacio de Loyola". Perú

Background: Neuroinflammation is an immune response of the Central Nervous System (CNS) from harmful stimuli appearing as a result of trauma, infection, or neurodegenerative diseases. Astrocytes are the main cells that contribute to the immune response. Excessive activation of astrocytes causes oxidative stress and activation of numerous responsable for triggering apoptotic neuronal death, which has been shown in neurodegenerative diseases such as Alzheimer disease (AD), Parkinson's disease (PD) molecular mechanisms, amyotrophic lateral sclerosis (ALS) and a number of other diseases of the nervous system. Epidemiological studies show the successful use of medicinal plants as an alternative treatment to various clinical conditions. It has been demonstrated that Uncaria tomentosa (UT) has a high antioxidant activity; anti-cancer, antiapoptotic and anti-inflammatory notably, both animal and human models.

Objectives: Evaluate the neuroinmuno modulating action of Uncaria tomentosa on astrocyte activity, in an animal model stimulated with LPS.

Methods: It was used for biological assays consistent commercial product in a hydroalcoholic 
extract of Uncaria tomentosa. The powdered extract was obtained using ethanol and water in $70 / 30$ by one hour at $20^{\circ} \mathrm{C}$, subsequently it is spray dried. Also, were used 40 albino rats Sprague-Dawley strain (240 - $260 \mathrm{~g}$ ). The coordinates were used in this study for the micro intracerebroventricular injection (ICV) were bregma: AP:- $0.9 \mathrm{~mm}$; L:1.8mm, V:3.8mm. Animals were treated prior to stereotactic surgery with Uncaria tomentosa $(5 \mathrm{mg} / \mathrm{ml}$ and $10 \mathrm{mg} / \mathrm{ml}$ ) administered orally with orogastric probes for 21 days. Then they were stimulated with $2.5 \mathrm{uL}$ of LPS, which was applied in the lateral ventricle. The animals were sacrificed 48 hours after the LPS applied for morphological evaluation.

Results: These results demonstrate the neuromodulatory activity Uncaria tomentosa in astrocytes and the effect on the central nervous system by inhibiting the production of inflammatory cytokines. An additional test would be measuring cytokine levels in blood flow to observe as their expression in the central nervous system and peripheral.

Conclusions: The results show that administration of UT attenuated glial activation in the hippocampus. The decrease is proportional to the dose of UT (100 $\mathrm{mg} / \mathrm{kg}$ or $500 \mathrm{mg} / \mathrm{kg}$ ) Model fits in the evaluation of CNSinflammation model.

\section{CARDIOMETABOLIC RISK RELATED TO CHRONOTYPE OF CHILDREN BETWEEN 6 TO 12 YEARS}

Baquerizo, $\mathbf{L}^{1}$, Trullén, $\mathbf{M}^{2}$, Santillán, $\mathbf{P}^{1}$, Simeón, A ${ }^{1}$, Lazo, P $^{1}$, Huamán, L ${ }^{3}$, Muñoz, $A^{4}$, Loayza, $\mathrm{L}^{5}$, Baquerizo, $\mathbf{M}^{1}$, Aguilar, $\mathbf{L a}^{4}$.

1. Laboratory of neuroscience and behavior- Cayetano Heredia Peruvian University. Peru.

2. University of Alfonso X "El Sabio". Spain.

3. Faculty of science and philosophy-Cayetano Heredia Peruvian University. Peru.

4. Faculty of Science of Health. University "San Ignacio de Loyola". Perú.

5. Deparment of Nutrition. University "San Ignacio de Loyola”. Perú.

Background: Alterations in normal sleep and wake rhythms directly influence the deterioration of health status and increase the risk of development of noncommunicable chronic diseases and associated comorbidities.

Objectives: The main objective was to determine the prevalence of factors associated with cardiometabolic risk according to the chronotype in children aged 6 to 12 years.

Methods: In this observational, descriptive, cross-sectional study, to determine the chronotype the Munich Chronotype Questionnaire (MCQ) was applied in an interview with 76 children, also anthropometric measurement (weight, height and abdominal perimeter) were measured. For the statistical analysis, the normality of the variables was checked. The variables were described as mean +/- standard deviation (ordinal variable) and $\mathrm{n}(\%)$ (categorical variable). To determine the differences between groups, a factorial ANOVA was performed between the chronotype categories according to middle sleep time, according to the authors' criteria based on similar studies, (Q1, Q2, Q3, Q4 and Q5) and the cardiovascular risk level (low, high and very high risk).

Results: The prevalence of overweight according to body mass index for age of $13 \%$ in men and $20 \%$ in women. The prevalence of cardiometabolic risk, according to abdominal perimeter for age, were low risk, high risk and very high risk of 50, 20 and 30\% in men and 50, 30 and $19 \%$ in women, respectively. No statistically significant differences were found in the prevalence of risk between groups according to MCQ variables, such as bedtime, sleep duration and chronotype; However, dividing the group into quintiles yielded a trend toward significance for the difference between groups. After, in the post hoc analysis no differences were found between the analyzed groups.

Conclusions: The phenotypic characteristics of the sample under study, as well as the related indexes, such as cardiovascular risk, do not show a relationship with the data derived from the MCQ. A large dispersion on data was found, which made it difficult to analyze and interpret the results. New lines of research, which may have a larger sample size, are necessary to elucidate the existing potential relationship between the aspects derived from the Munich chronotype and the nutritional status of a child population. 